Article accepted in Journal of Theoretical Biology

\section{Bifurcations analysis of oscillating hypercycles}

\author{
Antoni Guillamon ${ }^{1}$, Ernest Fontich ${ }^{2, *}$, Josep Sardanyés ${ }^{3,4, *}$ \\ 1. Departament de Matemàtica Aplicada, I EPSEB, Universitat \\ Politècnica de Catalunya, Av. Doctor Marañón, 44-50, 08028 \\ Barcelona, Spain \\ 2. Departament de Matemàtica Aplicada i Anàlisi, Universitat de \\ Barcelona, Gran Via de les Corts Catalanes 585, 08007 Barcelona, \\ Spain \\ 3. ICREA-Complex Systems Lab, Department of Experimental and \\ Health Sciences, Universitat Pompeu Fabra, Dr. Aiguader 88, 08003 \\ Barcelona, Spain \\ 4. Institut de Biologia Evolutiva (CSIC-Universitat Pompeu Fabra), \\ Passeig Maritim de la Barceloneta 37, 08003 Barcelona, Spain \\ * Corresponding authors. E. Fontich: fontich@ub.edu; J. \\ Sardanyés: josep.sardanes@upf.edu
}

\section{Abstract}

We investigate the dynamics and transitions to extinction of hypercycles governed by periodic orbits. For a large enough number of hypercycle species $(n>4)$ the existence of a stable periodic orbit has been previously described, showing an apparent coincidence of the vanishing of the periodic orbit with the value of the replication quality factor $Q$ where two unstable (non-zero) equilibrium points collide (named $Q_{S S}$ ). It has also been reported that, for values below $Q_{S S}$, the system goes to extinction. In this paper, we use a suitable Poincaré map associated to the hypercycle system, which is used to analyze the dynamics in the bistability regime, where both oscillatory dynamics and extinction are possible. The stable periodic orbit is identified, together with an unstable periodic orbit. In particular, we are able to unveil the vanishing mechanism of the oscillatory dynamics: a saddle-node bifurcation of periodic orbits as the replication quality factor, $Q$, undergoes a critical fidelity threshold, $Q_{P O}$. The identified bifurcation involves the asymptotic extinction of all hypercycle members, since the attractor placed at the origin becomes globally stable for values $Q<Q_{P O}$. Near the bifurcation, these extinction dynamics display a periodic remnant that provides the system with an oscillating delayed transition. Surprisingly, we found that the value of $Q_{P O}$ is slightly higher than $Q_{S S}$, thus identifying a gap in the parameter space where the oscillatory dynamics has vanished while the unstable equilibrium points are still present. We also identified a degenerate bifurcation of the unstable periodic orbits for $Q=1$.

\section{INTRODUCTION}

Hypercycles are catalytic networks with cyclic architecture. Hypercycle theory was developed as a biochemicallyplausible solution to the error catastrophe of error-prone, prebiotic replicators $[1,2]$. It has been theoretically conjectured that early replicators may have a limit in their length due to the expected large accumulation of errors during replication [1-5]. Eigen and Schuster proposed the hypercycle theory as a way to ensure the coexistence of distinct replicators able to store larger information allowing to surpass such an information constraint. It is known that hypercycles achieve coexistence of all replicators by means of bistability [1, 6-9]. Under bistability, large enough initial conditions of hypercycle elements allow the survival and persistence of all the catalytic species. On the contrary, low initial conditions involve the asymptotic extinction of the hyperycle since the dynamics are captured by a stable attractor found in the origin of the phase space. The dynamics of autocatalytic replicators $[10,11]$ and two-member hypercycles $[7,10]$ have been widely characterized. Hypercycles with three and four species display the same asymptotic behavior than smaller catalytic networks but the coexistence attractor is approached by means of damped oscillations [1,6]. Hypercycles with $n>4$ can undergo periodic oscillations due to the presence of attracting periodic orbits $[1,8,12-14]$.

A key aspect of hypercycles is the presence of bifurcations separating survival from extinction. Previous research has characterized these bifurcations in small hypercycles with replicators degradation $[6,7]$ and mutation $[14,15]$. The switch from the bistable to the monostable (i.e., onlyextinction) regimes in small hypercycles is usually governed by saddle-node bifurcations, which have been thoroughly characterized in small catalytic systems $[6,7,15]$. However, bifurcations in larger hypercycles governed by periodic orbits have not been explored. In this sense, the characterization of the nature of the transitions between survival and extinction in oscillating hypercycles is interesting to understand the dynamics of large, oscillating catalytic replicator networks. Such systems could store larger information contents, perhaps driving the transition from only RNA-based evolution to more sophisticated replicating systems coding for error-correction mechanisms.

A recent work developed by Silvestre and Fontanari [16] provided results on the dynamics characterizing the presence of periodic orbits governing the coexistence dynamics for hypercycles with $n \geq 5$ members in competition with a tail of mutants. These authors provided numerical evidence for the existence of a stable periodic orbit below a mutation threshold of the hypercycle elements. Also, they found an apparent coincidence between the value of mutation causing the global extinction of the hypercycle and the mutation value making two unstable fixed points colliding and becoming complex.

In this work we analyze the model in [16] focusing on the bifurcations driving towards extinction due to changes in mutation rates. By analyzing the dynamics using a Poincaré map, we show that the asymptotic extinction of oscillating hypercycles is given by a saddle-node bifurcation of periodic orbits, which is responsible for the shift separating the bistable from the monostable regimes. The article is organized as follows. First, we introduce the system under investigation and the mathematical model in Section IIA. Section IIB describes the symmetry of the system, also providing the stability analysis of the equilibrium points. Section III is devoted to the analysis of the periodic orbits and the bifurcation that involves 
the transition from the bistable to the monostable scenario. Specifically, we first give conditions for existence and location of periodic orbits. In Section IIIA we make a numerical prospection to find evidences of existence of a unique stable periodic orbit. Then, in Section IIIB we perform a numerical continuation of both stable and unstable periodic orbits and we describe the bifurcation that explains the vanishing of the periodic orbits for low values of the replication copying fidelity. Finally, Section IV is devoted to some conclusions.

\section{BASIC FACTS ON THE HYPERCYCLE MODEL}

\section{A. Mathematical model}

We consider the hypercycle system analyzed in [16] (see also [14]). The system is composed by $n$ functional templates with heterocatalytic connections and a pool of mutant sequences, which do not participate in the catalysis, thus acting as parasites $[9,14,17]$. The model is given by the following set of differential equations:

$$
\begin{aligned}
& \dot{x}_{i}=F_{i}(x):=x_{i}\left(A_{i} Q+K_{i} x_{i-1} Q-\phi\right), \\
& \dot{x}_{e}=x_{e}\left(A_{e}-\phi\right)+(1-Q) \sum_{i=1}^{n} x_{i}\left(A_{i}+K_{i} x_{i-1}\right),
\end{aligned}
$$

for $1 \leq i \leq n$ (we use the convention that $x_{0}=x_{n}$ ), where

$$
\boldsymbol{\phi}=\sum_{i=1}^{n} x_{i}\left(A_{i}+K_{i} x_{i-1}\right)+A_{e} x_{e} .
$$

Here $x_{i}$ are the population numbers of the functional hypercycle template $i$, while $x_{e}$ corresponds to the population numbers of the pool of mutants. Parameters $A_{i}$ and $Q$ are, respectively, the Malthusian (non-catalytic) replication rate of species $x_{i}$ and the average copying fidelity during replication (being $1-Q$ the average mutation rate). Constant $A_{e}$ is the Malthusian replication rate of the mutant sequences. Constants $A_{i}, Q, K_{i}$, and $A_{e}$ are positive. The term $\phi$ is a dilution outflow that keeps the total population constant and introduces competition between all the hypercycle members and the pool of mutants. The fact that the population is constant is expressed by the property that $\sum_{i=1}^{n} x_{i}+x_{e}=1$ is invariant. This permits to write $x_{e}$ in terms of $x_{i}$ and realize that therefore the dynamics of $x_{i}$ governed by (1) is independent of $x_{e}$. For that reason henceforth we focus on (1) which we consider in the bounded domain (simplex)

$$
\Omega=\left\{x \in \mathbb{R}^{n} \mid x_{i} \geq 0, \sum_{i=1}^{n} x_{i} \leq 1\right\}
$$

We denote by $F=\left(F_{1}, \ldots, F_{n}\right)$ the vector-field associated to (1). It is clear from the equations that the hyperplanes $\left\{x_{i}=0\right\}$ are invariant. Moreover, if $Q<1$, on $\sum_{i=1}^{n} x_{i}=1$, the vector-field points inwards $\Omega$. This is easily checked by computing $\vec{\nu} \cdot F(x)$, where $\vec{\nu}=(1, \ldots, 1)$ is a vector normal to the hyperplane $\sum_{i=1}^{n} x_{i}-1=0$. We have

$$
\vec{\nu} \cdot F(x)=(Q-1) \sum_{i=1}^{n} A_{i} x_{i}+(Q-1) \sum_{i=1}^{n} K_{i} x_{i} x_{i-1},
$$

which is strictly negative if $Q<1$. If $Q=1$ the hyperplane is invariant. These features imply that $\Omega$ is positively invariant.

\section{B. The symmetric hypercycle}

When $A_{i}=a$ and $K_{i}=k$, system (1) has a symmetry which helps to describe the dynamics. We take $A_{i}=A_{e}=$ $a>0$, and $k_{i}=1$ for $1 \leq i \leq n$, and we assume $Q \in(0,1]$. Then

$$
\phi=a+\sum_{i=1}^{n} x_{i} x_{i-1}
$$

Symmetry involves that the functional templates and parasites are selectively neutral, having the same kinetic properties [16]. To describe the symmetry of the system we introduce the linear map $R$ associated to the matrix

$$
R=\left(\begin{array}{ccccc}
0 & 0 & \ldots & 0 & 1 \\
1 & 0 & \ldots & 0 & 0 \\
0 & 1 & \ldots & 0 & 0 \\
\vdots & & & & \\
0 & 0 & \ldots & 1 & 0
\end{array}\right)
$$

We have that $R^{\top} R=\mathrm{Id}$, hence $R^{-1}=R^{\top}$. Moreover, $R^{n}=$ Id . The vector-field $F$ satisfies

$$
R \circ F=F \circ R,
$$

which implies that if $x(t)$ is a solution of $x^{\prime}=F(x)$, then $R x(t)$ is also a solution. Indeed,

$$
\dot{\overline{R x}}(t)=R \dot{x}(t)=R F(x(t))=F(R x(t)) .
$$

Moreover, if $\gamma(t)$ is a periodic orbit, $R \gamma(t)$ is also a periodic orbit and since $R$ is continuous (because it is linear) the stability character of both orbits is the same.

The equilibrium points of Eq. (1) are $X_{0}=(0, \ldots, 0)$ and $X_{ \pm}^{*}=\left(x_{ \pm}^{*}, \ldots, x_{ \pm}^{*}\right)$, where $x_{ \pm}^{*}$ are the solutions of $n x^{2}-$ $Q x-a(Q-1)=0$, that is:

$$
x_{ \pm}^{*}=\frac{Q \pm \sqrt{Q^{2}-4 n a(1-Q)}}{2 n} .
$$

Note that the equilibrium points $X_{ \pm}^{*}$ are real provided the condition

$$
Q^{2}-4 n a(1-Q) \geq 0
$$

holds. Otherwise, they live in the complex phase space.

The system has a critical copying fidelity value given by (see [16]):

$$
Q_{S S}=2(\sqrt{n a(1+n a)}-n a) .
$$


In [16], the authors claim that, for $Q<Q_{S S}$ the equilibrium points $X_{ \pm}^{*}$ vanish and the hypercycle becomes extinct, having a whole population of mutant sequences, i.e., $x_{e}=1$. The authors also identified the viability of the hypercycle depending on the number of functional templates, $n_{m}$, with

$$
n_{m}=\frac{Q^{2}}{4 a(1-Q)} \text {. }
$$

Similarly, a critical replication condition separating the viability of the hypercycle and the asymptotic extinction is given by

$$
a_{c}=\frac{Q^{2}}{4 n(1-Q)}
$$

For this latter case, if $a>a_{c}$ the hypercycle will be viable [16].

The equilibrium points $X_{ \pm}^{*}$ belong to the line $\left\{x_{1}=\cdots=\right.$ $\left.x_{n}\right\}$ which is the set of fixed points of $R$. The conjugation condition (3) implies, taking derivatives, that $R \cdot D F=D F \circ$ $R \cdot R$ and hence at $X_{ \pm}^{*}$ we have

$$
R D F\left(X_{ \pm}^{*}\right)=D F\left(X_{ \pm}^{*}\right) R .
$$

This property implies that $D F\left(X_{ \pm}^{*}\right)$ is a so-called circulant matrix, i.e., a matrix of the form

$$
\left(\begin{array}{cccc}
c_{1} & c_{2} & \ldots & c_{n} \\
c_{n} & c_{1} & \ldots & c_{n-1} \\
\vdots & & \ddots & \vdots \\
c_{2} & c_{3} & \ldots & c_{1}
\end{array}\right)
$$

For these matrices there exist formulas allowing to compute all the eigenvalues and eigenvectors [18]. In fact, the eigenvalues are given by the discrete Fourier transforms of the sequence $\left\{c_{k}\right\}$, see [18, Sect. 3.1]:

$$
\psi_{m}=\sum_{k=1}^{n} c_{k} e^{-2 \pi i(m-1)(k-1) / n}, \quad 1 \leq m \leq n .
$$

In particular, the Jacobian matrix at the critical point $X_{0}$ is $a(Q-1) \mathbf{I}_{n}$, being $\mathbf{I}_{n}$ the identity matrix. This means that $X_{0}$ is stable whenever $Q<1$ and becomes unstable for $Q>1$; the bifurcation occurring at $Q=1$ is of codimension $n$ since all the eigenvalues change sign.

On the other hand, the Jacobian matrix at the critical points $X_{ \pm}^{*}$ is given by the matrix (5) with $c_{1}=a(Q-1)+Q x_{ \pm}^{*}-$ $(n+2)\left(x_{ \pm}^{*}\right)^{2}=-2\left(x_{ \pm}^{*}\right)^{2}$ (here we apply the definition of $\left.x_{ \pm}^{*}\right), c_{j}=-2\left(x_{ \pm}^{*}\right)^{2}$, for $j=2, \ldots, n-1$, and $c_{n}=x_{ \pm}^{*}(Q-$ $\left.2 x_{ \pm}^{*}\right)$.

Straightforward computations using properties of the roots of unity lead to the following expressions of the eigenvalues,

$\psi_{1}=x_{ \pm}^{*}\left(Q-2 n x_{ \pm}^{*}\right), \quad \psi_{j}=Q x_{ \pm}^{*} e^{2 \pi i(j-1) / n}, j=2 \div n$

thus showing a nice geometrical structure: all the eigenvalues but one lie on the circle of radius $Q x_{ \pm}^{*}$ in the complex plane at angles corresponding to the roots of unity, except for the first root of unity $e^{2 \pi i 0}=1$. Instead, we have the eigenvalue $\psi_{1}=$ $x^{*}\left(Q-2 n x^{*}\right)$ which is negative (resp., positive) for $x^{*}=x_{+}^{*}$ (resp., for $x^{*}=x_{-}^{*}$ ). Observe also that for $n<4, X_{+}^{*}$ (resp., $X_{-}^{*}$ ) is always stable (resp., unstable), whereas for $n=4$ (and multiples of 4), a central manifold for these points arises since two of the eigenvalues are purely imaginary. For $n>5$, both $X_{+}^{*}$ and $X_{-}^{*}$ have eigenvalues with both positive and negative real parts. In this paper we will restrict ourselves to the case $n=5$, in which $X_{+}^{*}$ has 3 eigenvalues with negative real part and 2 eigenvalues with positive real part, which implies a three-dimensional stable manifold and a two-dimensional unstable manifold; for $X_{-}^{*}$ these dimensions are swapped. See also [21] for a similar study in a related system.

\section{PERIODIC ORBITS}

Numerical simulations indicate that, for $Q>Q_{S S}$, Eq. (1) with $i \geq 5$ has one and just one asymptotically stable periodic orbit $\gamma(t)$, see [16]. By the symmetry property, $\gamma$ has to be mapped to itself by $R$ so that $R \gamma(0)=\gamma(\tau)$ for some $\tau$. Since $R \gamma(t)$ and $\gamma(t+\tau)$ satisfy the same initial value problem, by uniqueness of solutions we obtain that

$$
R \gamma(t)=\gamma(t+\tau), \quad t \in \mathbb{R} .
$$

This means that $\gamma_{j}(t)=\gamma_{j+1}(t+\tau)$ for all $t, 1 \leq j \leq n-1$, and therefore

$$
\gamma_{j}(t)=\gamma_{n}(t+(n-j) \tau), \quad 1 \leq j \leq n,
$$

which means that all components of the periodic orbit can be obtained from the last one with a shift of time. We also have $R^{k} \gamma(t)=\gamma(t+k \tau)$. In particular, when $k=n$, $\gamma(t)=\gamma(t+n \tau)$ which shows that the period $T$ is related to $\tau$ by $T=n \tau$. This result can be observed in Fig. 1.B4, which displays the dynamics of all hypercycle members in the coexistence scenario, which oscillate following the same pattern but shifted in time.

Our aim is to understand how the periodic orbits arise/appear in terms of the parameter $Q$ by means of accurate numerical computations. For this purpose, it is very important to locate the periodic orbits when they exist. For that we introduce

$$
\Sigma_{\alpha}=\left\{x \in \Omega \mid \sum_{j=1}^{n} x_{j}=\alpha\right\}, \quad 0<\alpha \leq 1 .
$$

Let $\vec{\nu}=(1, \ldots, 1)$ be a normal vector to $\Sigma_{\alpha}$. The sign of the scalar product $\vec{\nu} \cdot F(x)$ is related with the angle of $\vec{\nu}$ and the vector-field at $x$. In particular, if $\vec{\nu} \cdot F(x)<0$ for all $x \in \Sigma_{\alpha}$ we have that all solutions that arrive at $\Sigma_{\alpha}$ cross from $\sum_{j=1}^{n} x_{j}>\alpha$ to $\sum_{j=1}^{n} x_{j}<\alpha$. We have

$\vec{\nu} \cdot F(x)=-a(1-Q) \sum_{j=1}^{n} x_{j}-\left(\sum_{j=1}^{n} x_{j}-Q\right) \sum_{j=1}^{n} x_{j} x_{j-1}$.

Then, if $Q \leq \alpha<1$ and $x \in \Sigma_{\alpha}, \vec{\nu} \cdot F(x) \leq-a(1-Q) \alpha<0$. If $\alpha<Q$, using that the maximum of $\sum_{j=1}^{n} x_{j} x_{j-1}$ on $\Sigma_{\alpha}$ is 

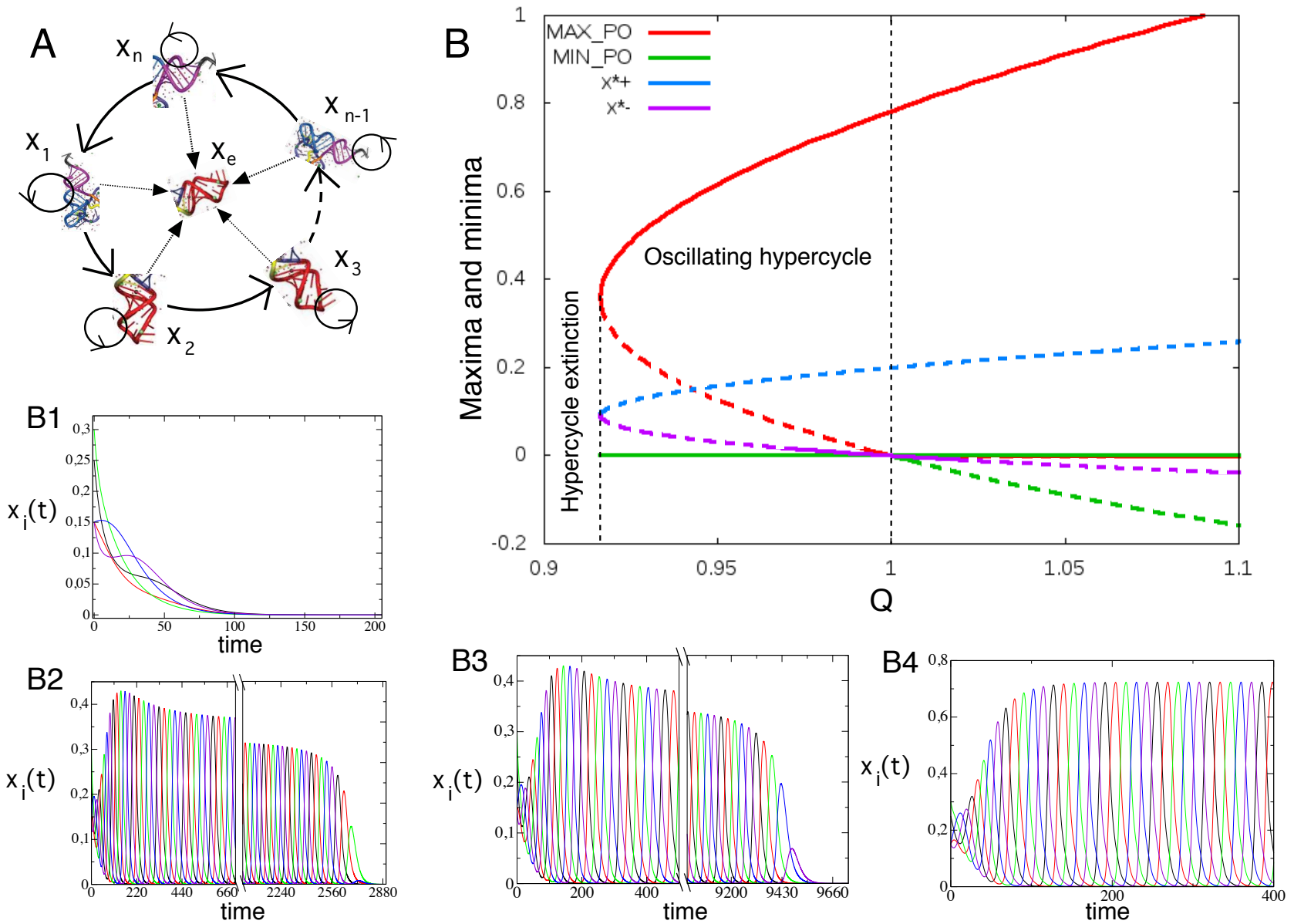

FIG. 1: (A) Hypercycle of $n$ members with an error tail of mutants. Notice that the hypercycle members $\left(x_{i}\right)_{i=1, \ldots, n}$, can produce a noncatalytic mutant $\left(x_{e}\right)$ during replication, which competes with the catalytic cycle (i.e., the mutant does not provide catalytic aid to any hypercycle species behaving as a parasite). (B) Bifurcation diagram tuning the replication quality factor $Q$, using $a=0.5$ and $n=5$. For this parameter combination, the saddle-node bifurcation of the unstable fixed points takes place at $Q_{S S}=0.916079783$. We also identified a saddle-node of periodic orbits taking place at $Q_{P O}=0.916139611$, and a degenerate bifurcation of unstable periodic orbits and $X_{0}$ taking place at $Q=1$. Notice that there is a gap between the bifurcation value $Q_{S S}$ and $Q_{P O}$ (see Fig. (2)). The stable and unstable equilibria are shown with solid and dashed lines, respectively. The time series display the dynamics for different values of $Q$ with: (B1) $Q=0.87<Q_{S S}$; (B2) $Q=0.916078 \lesssim Q_{S S}$; (B3) $Q_{S S} \lesssim Q=0.916135 \lesssim Q_{P O}$; (B4) $Q_{P O}<Q=0.98$ (each color represents the concentration of each hypercycle member). See movie.avi for an animation of the bifurcations in panel B.

$\alpha^{2} / 4$, we have

$$
\vec{\nu} \cdot F(x)<\frac{\alpha}{4}\left(-\alpha^{2}+Q \alpha-4 a(1-Q)\right) .
$$

Let $\alpha_{0}=0$ and $\alpha_{ \pm}=\left(Q \pm \sqrt{Q^{2}-16 a(1-Q)}\right) / 2$ be the zeros of the previous expression. Let $Q_{*}=-8 a+$ $\sqrt{64 a^{2}+16 a}$. If $Q>Q_{*}$ then $\vec{\nu} \cdot F(x)<0$ on $\Sigma_{\alpha}$ for $\alpha \in\left(0, \alpha_{-}\right) \cup\left(\alpha_{+}, Q\right)$. Observe that $Q_{*}=\left.Q_{S S}\right|_{n=4}$. As a consequence of the previous calculations we have

- if $Q<Q_{*}$, the full domain $\Omega$ is contained in the basin of attraction of the origin. Therefore there are not periodic orbits.

- if $Q \geq Q_{*}$, the periodic orbits, if they exist, are contained in $\left\{x \in \Omega \mid \alpha_{-} \leq \sum_{j=1}^{n} x_{j} \leq \alpha_{+}\right\}$.
Another consequence of these calculations is the proof for the case $n \leq 4$ of a conjecture by Silvestre and Fontanari [16] which states that if Eq. (1) has a stable periodic orbit then it has an unstable equilibrium point. Indeed, Eq. (1) has not the unstable equilibrium point if and only if $Q^{2}<4 n a(1-Q)$. For $n \leq 4$, the previous condition becomes $Q^{2}<16 a(1-Q)$ and hence $\alpha_{ \pm}$are complex, which means that there can not be a periodic orbit. Notice however that our computations described in IIIB show that they may exist an unstable fixed point without the stable periodic orbit.

\section{A. Computation of periodic orbits}

From a numerical point of view, to find asymptotically stable periodic orbits is as simple as taking an initial condition 

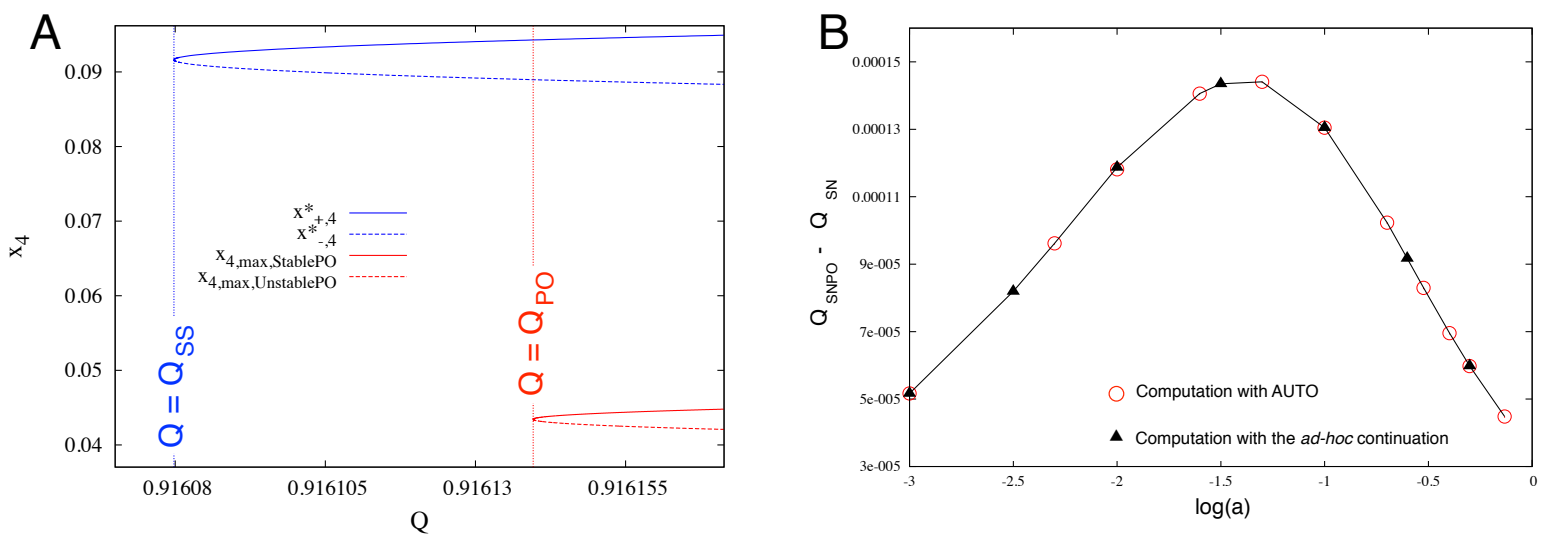

FIG. 2: (A) Bifurcation diagram with respect to $Q$ for both the fixed points and the periodic orbits for $a=0.5$. (B) Gap between the two saddle-node bifurcations as a function of the parameter $a$; namely, the gap between saddle-node of critical points, indicated by $Q_{S S}:=$ $2(\sqrt{(n a(1+n a))}-n a)$, and the saddle-node of periodic orbits, $Q_{P O}$, which has been computed numerically. For each value of $a$, we have performed the numerical computation of the $Q_{P O}$ by means of an ad-hoc program that uses the continuation method with high-precision (black triangles), as explained in the Appendix A.3. Additionally, for the sake of completeness and consistency, we have computed the same bifurcation points using the software AUTO (red circles). While the gap between the two bifurcation points is of the order $10^{-4}$, we found the difference between the two numerically computed values of $Q_{P O}$ to be of order $10^{-8}$, as evidenced in the figure, where the two sets of values lie on the same interpolating lines.

on its basin of attraction and integrate the equation forward: after some transient, the trajectory will be very close to the periodic orbit. However, this method does not apply to find unstable periodic orbits. Since our goal is understanding the bifurcations undergone by all periodic orbits, we have computed the Poincaré map $P$ associated to a suitable transversal section $\Sigma$ (see Appendix A.1), a tool that allows to deal both with stable and unstable periodic orbits. In Fig. 3A we show the transversal section $\Sigma$ together with the unstable and the stable periodic orbits for a specific value of the parameters, in the subspace $\left(x_{1}, x_{4}, x_{5}\right)$. We have that periodic orbits of the vector-field correspond to fixed or periodic points of the map $P$. The Poincaré map $P$ can be simulated numerically using an integration method for ODEs with initial condition $x \in \Sigma$, integrating up to the solution reaches $\Sigma$ again, crossing it in the same direction. Then, fixed points of $P$ are obtained applying Newton's method to either the function $f(x)=P(x)-x$ for fixed points or $f(x)=P^{k}(x)-x$ for $k$-periodic points of $P$. In Fig. 3B we show projections on the $\left(x_{4}, x_{5}\right)$ subspace of the iterations of the Poincare map on the transversal section $\Sigma$; they tend either to the stable periodic orbit (blue iterates) represented by a fixed point $X^{s} \in \Sigma$ of $P$ or to the unstable one (black iterates, zoomed out on the lower left corner), represented by a fixed point $X^{u} \in \Sigma$ of $P$. Fig. $3 \mathrm{C}$ shows different iterates tending to $X^{s}$, for different values of the copying fidelity $Q$, of the Poincaré map restricted to $\Sigma$.

As a first exploration, in order to check that there exists a unique stable periodic orbit inside the simplex $\Omega$, we performed a simple numerical experiment consisting of a MonteCarlo (MC) algorithm. We fixed the set of parameter values to be $a=0.001, K=1$, and $Q=0.2>Q_{S S}$ for which we expect there exists a stable periodic orbit. Next, we identified the fixed point $z^{*} \in \Sigma$ of the Poincaré map corresponding to a stable periodic orbit (see Appendix A. 2 for a detailed description of the MC algorithm) and we defined a ball of radius $\epsilon=10^{-8}$ around $z^{*}$. We then took a sampling of $N=5 \times 10^{7}$ random initial conditions within the simplex and integrated the corresponding trajectories over 35000 time units. Finally, we checked whether the intersection of the trajectory on the Poincaré section $\Sigma$ laid within this ball or radius $\epsilon$. Otherwise, we considered the periodic orbit as different from the one corresponding to $z^{*}$, including the case when the orbit goes to 0. The results of the simulations revealed that among all random initial conditions: (i) all of them cut the Poincaré section, and (ii) all of them ended up either to 0 or within the radius around the fixed point of the Poincare section. Then, we provide a numerical evidence about the existence and uniqueness of a stable periodic orbit.

This MC procedure was systematically replied on a grid of $31 \times 31$ points in the parameter region $\{(a, Q) \mid a \in$ $[0.01,0.5], Q \in[0.2,0.99]\}$. For each pair of values of the parameters we took $N=10^{4}$ random points within the simplex as initial conditions of the Newton method to find fixed points of the Poincare map $P$. In all cases we have found convergence to a unique stable periodic orbit.

\section{B. Bifurcation analysis}

We have applied a continuation method for periodic orbits to obtain the respective bifurcation diagram. This is a key point in the paper since we have been able not only to de- 

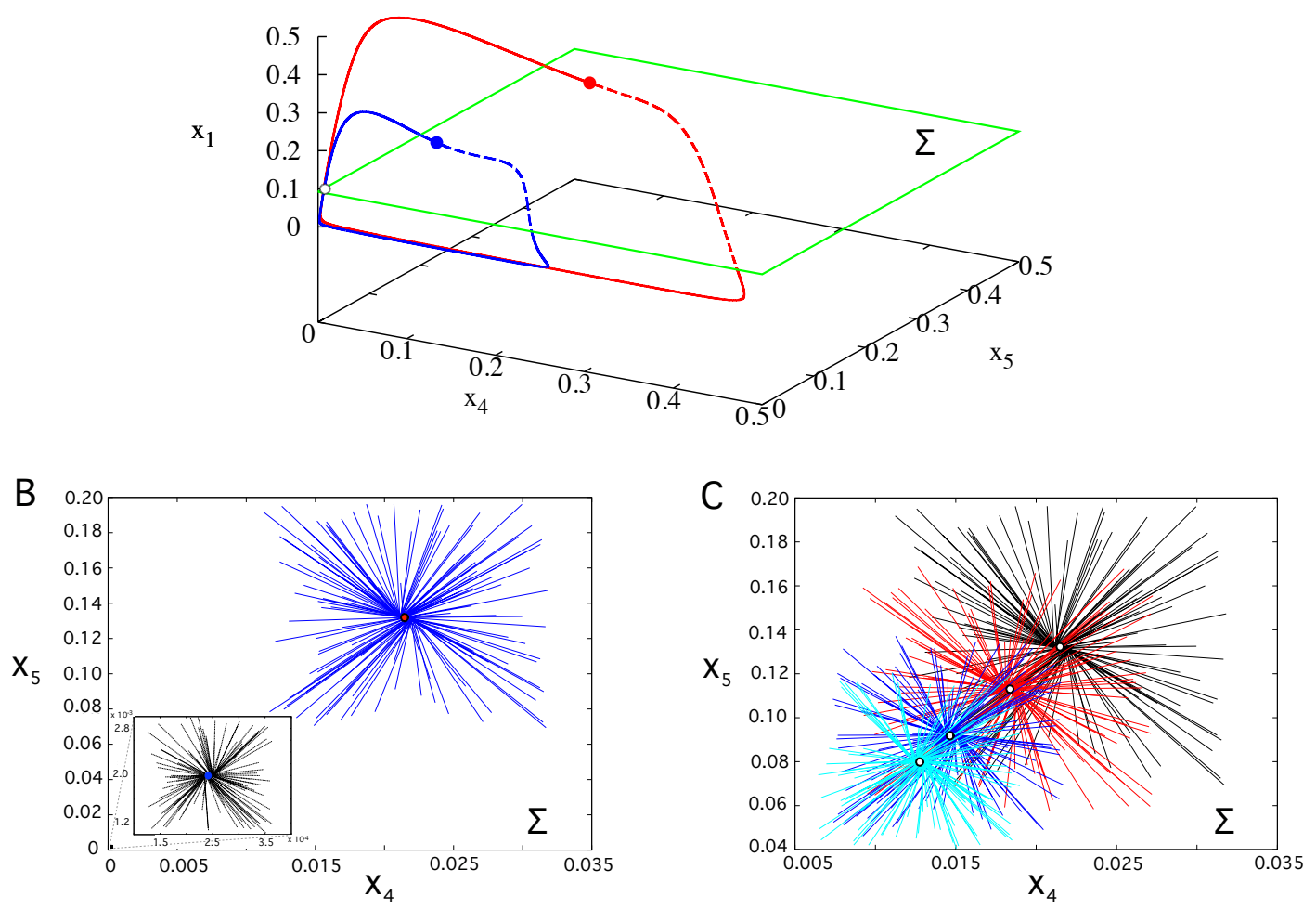

FIG. 3: (A) Representation of the Poincaré section, $\Sigma$, used to characterize the periodic orbits and the bifurcation of the system. (B) Iterates of the Poincaré map using $a=0.5, Q=0.2$ and $n=5$. The main plot displays several iterates converging to the equilibrium of the Poincaré map corresponding to the stable periodic orbit. The inset shows an enlarged view of several iterates converging to the fixed point of the map corresponding to the unstable periodic orbit, responsible of the saddle-node bifurcation with the stable periodic orbit. (C) Location of the fixed point of the stable periodic orbit on the Poincaré section by decreasing $Q$, with $Q=0.2$ (black); $Q=0.18$ (red); $Q=0.16$ (blue); $Q=0.154$ (cyan). The open circles correspond to the fixed point of the Poincaré map. Note that as $Q$ diminishes, this fixed point travels towards the unstable periodic orbit, which is closeer to the point $(\mathbf{0})$.

scribe the bifurcations but also to detect a gap between the periodic orbit and the fixed point bifurcations that was not detected in the previous literature. The continuation method (see Appendix A.3. for details) consists of identifying the periodic orbits with zeros of an implicit equation and thus implementing a procedure of continuation of zeros. The most technical challenge in the method is tracking the bifurcation curves at the turning points as the ones encountered in saddle-node bifurcations $\left(Q_{P O}\right)$. In such case we assume that the zeros lie on a parameterized curve $s \mapsto(x(s), \lambda(s))$ and we perform the continuation with respect to the parameter $s$.

With this method we are able to find a discretization of the curves and confirm the saddle-node bifurcation of periodic orbits with respect to the copying fidelity parameter $Q$; that is, we prove the existence of both a stable and an unstable periodic orbit for $Q>Q_{P O}$ that collide for $Q=Q_{P O}$ and disappear for lower values of $Q$. The bifurcation diagram is shown in Fig. 1B, where we plot the maximum and the minimum of both stable and unstable periodic orbits and the values of $x_{ \pm}^{*}$ for a 5 -membered hypercycle. Specifically, we use $a=0.5$, and, as the control parameter $(Q)$ decreases, both periodic orbits and the fixed points $x_{ \pm}^{*}$ approach to each other in the phase space, colliding at $Q=Q_{P O}$ and $Q=Q_{S S}$, respectively. The bifurcation diagram also reveals a degenerate bifurcation at $Q=1$. However, this value of $Q$ may correspond to a hypercycle with no mutation during replication, which, from a biological point of view, may be improbable in hypercycles.

The dynamics of the hypercycle with $n=5$ species is displayed for different values of the bifurcation parameter $Q$. Figure 1.B1 displays the extinction scenario for $Q=$ $0.87 \lesssim Q_{S S}$, where all member hypercycles become rapidly extincted. If $Q$ approaches from below to $Q_{S S}$, the hypercycle also asymptotically extincts, but, since we are near the saddle-node bifurcation, transients become longer. This case is displayed in Fig. 1.B2, with $Q=0.916078<Q_{S S}$. Here, all hypercycle members oscillate for a long period of time before collapsing, in what is known as a delayed transition. Such a dynamics, which arises near saddle-node bifurcations, have been characterized in hypercycles [6, 7, 9, 10, 15, 19], in autocatalytic replicators $[10,11]$, as well as in singlespecies dynamics under Allee effects [20]. Interestingly, the 
same dynamics is found below the saddle-node bifurcation of fixed points and below the saddle-node of periodic orbits (Fig. 1.B3). When $Q>Q_{P O}$ the hypercycle survives since trajectories are attracted by the stable periodic orbit.

As we previously mentioned, we identified a gap in the parameter space tuning $Q$, used to build the bifurcation diagram. A zoom of this gap can be seen in (see Fig. 2A). Finally, Fig. 2B shows the dependence of the size of the gap on the parameter $a$. Here we show the distance computed with our computation method and the software AUTO (see [22]), used to test/compare the results obtained with the continuation method.

Figure 3A displays the Poincaré section, $\Sigma$ (green), which is cut by the stable (red) and unstable (blue) periodic orbit. In Fig. 3B we plot several trajectories that approach to the unstable and the stable periodic orbits depending on the initial conditions. Finally, in Fig. 3C we show how the fixed point of the stable periodic orbit in the Poincaré section approaches towards the fixed point of the unstable orbit (not shown) in $\Sigma$ as $Q$ diminishes towards the bifurcation value $Q_{P O}$.

\section{CONCLUSIONS}

In this article we have investigated the bifurcations causing the asymptotic extinction of oscillating hypercycles using the model by Silvestre and Fontanari [16] (see also [14]). This model explored a hypercycle composed by $n$ catalytic templates with exponential and heterocatalytic replication competing with the so-called error tail. These authors provided numerical evidence for the existence of a stable periodic orbit below a mutation threshold of the hypercycle elements, in agreement with previous investigations [1, 8, 12-14]. They also identified the vanishing of the periodic orbit taking place below a critical value $Q_{S S}$ of the copying fidelity $Q$. Specifically, they found that the region of viability in the space $(Q, a)$, where the hypercycle has nonzero concentration, either in the static regime or in the periodic orbit condition, was determined by the condition of existence of real equilibrium points. They actually stated: "The periodic solution disappears at $Q \approx 0.19639$, the value at which the condition (4) for the existence of a nontrivial equilibrium is violated. We have no proof for this remarkable coincidence" [16].

We here investigate this phenomenon focusing on a 5member hypercycle, which is the minimal hypercycle size undergoing periodic oscillations. We show, by means of analytical and numerical investigations, that a saddle-node bifurcation of periodic orbits takes place at $Q=Q_{P O}$, being responsible of the change from the bistable to the monostable (global extinction) states. Saddle-node bifurcations are typically responsible of asymptotic extinction in catalytic networks. These bifurcations, which have been identified in small hypercycles $[7,9,15]$ as well as in autocatalytic replicators $[10,11]$, are known to separate the survival from the extinction phases.

Interestingly, we found that the bifurcation value responsible for the saddle-node bifurcation of periodic orbits slightly differed from the bifurcation value (i.e., $Q_{S S}$ ) at which the collision of two unstable fixed points takes place. Hence, the bifurcation value involving the collision of both stable and unstable periodic orbits does not coincide with the value of $Q$ limiting the existence of the equilibrium points reported in [16].

\section{Acknowledgements}

This work has been partially funded by the Botín Foundation (by Banco Santander through its Santander Universities Global Division) (JS), by the Spanish Grant Mineco MTM2013-41168-P (EF), by the Catalan Grant AGAUR 2014 SGR 1145 (EF), and by the MINECO Grant MTM201231714, and the Catalan Grant AGAUR 2014SGR-504 (AG).

\section{Appendix}

In the following appendices, we provide descriptions of the methods and algorithms used in the analyses of the periodic orbits and their bifurcations.

\section{A.1. Poincaré maps}

To compute periodic orbits we have used the device of looking for appropriate Poincaré maps and then search for fixed points of them.

Given a differential equation $\dot{x}=F(x), x \in \mathbb{R}^{n}$, a Poincaré section is a $(n-1)$-dimensional surface $\Sigma$ such that for all $x \in \Sigma, F(x)$ does not belong to the tangent $(n-1)$ plane of $\Sigma$ at $x$, so that the solutions cross transversally $\Sigma$ whenever they reach $\Sigma$. The Poincaré map (associated to $\Sigma$ ) sends a point $y$ in $\Sigma$ to the next intersection of the solution with initial condition $y$ with $\Sigma$, crossing $\Sigma$ in the same direction as in $y$ and provided it exists. It turns out that periodic solutions of $\dot{x}=F(x)$ correspond to fixed (or periodic) points of $P$. See for instance [23] for more details. Poincaré maps are effectively computed by using numerical integrators of differential equations.

\section{A.2. Algorithm for finding stable periodic orbits}

We developed a MonteCarlo (MC) exploration of the simplex by taking $N$ random initial conditions obtained from a uniform distribution, i.e., $x_{i}(0) \in U(0,1)$. The chosen initial conditions are imposed to fulfill the following conditions. The first condition involves that $\sum_{i} x_{i}(0) \leq 1$ and ensures that the initial conditions are inside the simplex $\Omega$. The second one is to discard a set of initial conditions that will asymptotically reach zero. Extinction is always possible because the equilibrium point $X_{0}=(\overrightarrow{0})$ is locally asymptotically stable. In order to estimate the initial conditions flowing to this attractor, we analyze the basin of attraction of $X_{0}$. We assume $Q<1$. Let $\mathcal{B}\left(X_{0}\right)$ denote the basin of attraction of 
the equilibrium point $X_{0}$. By the symmetry, if $x \in \mathcal{B}\left(X_{0}\right)$, $R x \in \mathcal{B}\left(X_{0}\right)$. We claim that

$$
\Omega \cap\left\{x \in \mathbb{R}^{n} \mid \exists j \text { such that } x_{j}=0\right\} \subset \mathcal{B}\left(X_{0}\right) .
$$

Indeed, by the symmetry it is enough to check that

$$
\begin{aligned}
\Omega_{n} & =\Omega \cap\left\{x \in \mathbb{R}^{n} \mid x_{n}=0\right\} \\
& =\left\{\left(x_{1}, \ldots, x_{n-1}, 0\right) \mid \sum_{i=1}^{n-1} x_{i} \leq 1\right\} \subset \mathcal{B}\left(X_{0}\right) .
\end{aligned}
$$

On $\Omega_{n}$, if $x_{1}>0, \dot{x}_{1}=x_{1}\left(a(Q-1)-\sum_{j=2}^{n-1} x_{j} x_{j-1}\right)<0$. Then, for any solution in $\Omega_{n}, x_{1}(t)$ decreases monotonically and converges to zero. Therefore, there exists $t_{1}$ such that if $t>t_{1}, x_{1}(t)<a(1-Q) / Q$. From $\dot{x}_{2}=x_{2}(a(Q-1)+$ $\left.Q x_{1}-\sum_{j=2}^{n-1} x_{j} x_{j-1}\right)$ we have that if $t>t_{1}, x_{2}(t)$ decreases monotonically also converging to zero. Then there exists $t_{2}$ such that if $t>t_{2}, x_{2}(t)<a(1-Q) / Q$. Proceeding in this way we get that $x_{j}(t)$ converges to zero, $1 \leq j \leq n-1$, which proves the claim. Then, $\mathcal{B}\left(X_{0}\right)$ is an open set that contains $\cup_{j=1}^{n} \Omega_{j}$. Analogous computations lead to prove that

$$
\left\{x \in \mathbb{R}^{n} \mid 0 \leq x_{j} \leq a(1-Q) / Q, 1 \leq j \leq n\right\} \subset \mathcal{B}\left(X_{0}\right) .
$$

So we can discard the random initial conditions in this set. Once an initial condition is determined, we integrate Eqs. (2) for $t=35,000$ (to ensure that we run at least several periods) using this initial condition. After this time, we check if the trajectory cuts the Poincaré section (see Section A.1.). If this is the case, we consider this intersection as a very good approximation of a point on a stable periodic orbit which is a fixed point of the Poincaré map. We define a ball of radius $\epsilon=10^{-8}$ around this fixed point. All of the initial conditions falling within this radius are considered to belong to the same stable periodic orbit. Possible points in the Poincare section found outside this radius are considered different stable periodic orbits. We repeat this process a certain number of times.

\section{A.3. Continuation method for periodic orbits}

Given a family of differential equations $\dot{x}=F(x, \lambda)$, $x \in \mathbb{R}^{n}$, depending on one parameter $\lambda \in \mathbb{R}$ for which we know a periodic orbit for a value of the parameter $\lambda=\lambda_{0}$, the continuation method permits to compute periodic orbits for different values of $\lambda$ whenever they exist.

Periodic orbits of $\dot{x}=F(x, \lambda)$ correspond to fixed points of a suitable Poincaré map $P_{\lambda}$ (or of an iterate of it, say $P_{\lambda}^{m}$ with $m \geq 1)$. Equivalently they correspond to zeros of $G(x, \lambda)=$ $P_{\lambda}^{m}(x)-x$. Then we can rephrase the problem as a problem of continuation of zeros of $G(x, \lambda)$.

In the simplest continuation method we assume we have a zero $x_{0}$ of $G$ when $\lambda=\lambda_{0}$, i.e., $G\left(x_{0}, \lambda_{0}\right)=0$. We choose a continuation step $\Delta \lambda$ and we define $\lambda_{k}=\lambda_{0}+k \Delta \lambda$. In the $k$-th step of continuation we look for $x_{k}$ such that $G\left(x_{k}, \lambda_{k}\right)=0$ using an iterative method for finding zeros (for instance, Newton's method) with initial guess $x=x_{k-1}$, i.e., the zero obtained in the previous step.

This method has the disadvantage that it can not go over turning points as the ones encountered in saddle-node bifurcations. In such case we assume that the zeros of $F$ are on a parameterized curve $s \mapsto(x(s), \lambda(s))$ and we perform continuation with respect to the parameter $s$ to find a discretization of such curve. Similarly as before, we assume that for $s=s_{0}$ we have $x_{0}=x\left(s_{0}\right), \lambda_{0}=\lambda\left(s_{0}\right)$ such that $G\left(x_{0}, \lambda_{0}\right)=0$, and we choose a continuation step $\Delta s$.

In the $k$-th step we have $x_{k-1}=x\left(s_{k-1}\right), \lambda_{k-1}=\lambda\left(s_{k-1}\right)$ such that $G\left(x_{k-1}, \lambda_{k-1}\right) \approx 0$ and we look for $\left(x_{k}, \lambda_{k}\right)=$ $\left(x\left(s_{k}\right), \lambda\left(s_{k}\right)\right)$ satisfying $G(x, \lambda)=0$.

First, we compute a prediction of $\left(x_{k}, \lambda_{k}\right)$ imposing that it should be close to the tangent line of the curve $(x(s), \lambda(s))$ at $s=s_{k-1}$ provided $\Delta s$ is small. To compute a tangent vector to the curve we differentiate

$$
G_{i}(x(s), \lambda(s))=0, \quad 1 \leq i \leq n,
$$

with respect to $s$, to obtain

$\sum_{j=1}^{n} D_{x_{j}} G_{i}(x(s), \lambda(s)) \dot{x}_{j}(s)+D_{\lambda} G_{i}(x(s), \lambda(s)) \dot{\lambda}(s)=0$,

with $1 \leq i \leq n$, which indicates that $\left(\dot{x}_{s}, \dot{\lambda}_{s}\right)$ has to be orthogonal to the vectors $\left(D_{x_{1}} G_{i}, \ldots, D_{x_{n}} G_{i}, D_{\lambda} G_{i}\right), 1 \leq$ $i \leq n$, evaluated at $(x(s), \lambda(s))$. This condition determines uniquely the direction, which is generated by $v=$ $\left(v_{0}, v_{1}, \ldots, v_{n}\right)$, where $v_{i}$ is $(-1)^{i}$ times the determinant of the submatrix of $D G(x, \lambda)$ obtained by removing the $i$-column. Then we make the prediction

$$
\left(x_{k}, \lambda_{k}\right)=\left(x_{k-1}, \lambda_{k-1}\right)+\frac{v}{\|v\|} \Delta s,
$$

and we use a method for optimization (for instance, least squares) to find the closest point to the computed prediction in $G(x, \lambda)=0$.

We have used the following iterative scheme to approach such point

$$
\xi_{l}=\xi_{l-1}-D G\left(\xi_{l-1}\right)\left(D G\left(\xi_{l-1}\right) D G\left(\xi_{l-1}\right)^{\top}\right)^{-1} G\left(\xi_{l-1}\right),
$$

with $l \geq 1$, where $\xi_{l}=\left(x_{l}, \lambda_{l}\right)$. See [24] for more details.

\section{A.4. Numerical tools}

Numerical integrations have been carried out with a Runge-Kutta-Fehlberg RKF78 method, with automatic step size control (using a step size $10^{-4} \leq \Delta t \leq 10^{-1}$ ) and the local relative tolerance $10^{-14}$

When applying Newton method for the Poincaré map we ask for an accuracy of $\epsilon=0.5 \times 10^{-14}$. The Jacobian matrix of the Poincaré map required by the Newton's method is computed using lateral derivatives with $h=10^{-5}$.

The continuation step was chosen to be $\Delta s=$ $\max \left(10^{-6}, 10^{-2}\left(Q-Q_{S S}\right)\right)$. In the iterative scheme (6), we 
obtain $\xi_{l}-\xi_{l-1}$ by solving a linear system by means of the LU method. Pivoting elements are required to be bigger than
$0.5 \times 10^{-10}$.
[1] Eigen M, Schuster P. The hypercycle. A principle of natural self-organization. Springer-Verlag (1979)

[2] Maynard-Smith, J., Szathmary, E. The Major Transitions in Evolution. W.H. Freeman-Spektrum (1995)

[3] Eigen, M. Self-organization of matter and the evolution of biological macromolecules. Naturwissenschaften 58, 465-523 (1971)

[4] Küppers, B-O. Molecular theory of evolution. Chapter: The information threshold. Springer-Verlag (1985)

[5] Swetina, J., Schuster, P. Self-replication with errors. A model for polynucleotide replication. Biophys. Chem. 16, 329 (1982)

[6] Sardanyés, J., Solé, R.V. Delayed transitions in non-linear replicator networks: About ghosts and hypercycles. Chaos, Solitons \& Fractals 31, 305-315 (2007)

[7] Sardanyés, J., Solé, R.V. Bifurcations and phase-transitions in spatially extended two-member hypercycles. J. Theor. Biol. 243, 468-482 (2006)

[8] Sardanyés, J. Ghosts in high-dimensional nonlinear dynamical systems: The example of the hypercycle. Chaos, Solitons \& Fractals 39, 92-100 (2007)

[9] Sardanyés, J., Solé, R.V. Spatio-temporal dynamics in simple asymmetric hypercycles under weak parasitic coupling. Physica D 31, 116-129 (2007)

[10] Sardanyés, J., Solé, R.V. The role of cooperation and parasites in non-linear replicator delayed extinctions. Chaos, Solitons \& Fractals 31, 1279-1296 (2007)

[11] Fontich, E., Sardanyés, J. General scaling law in the saddlenode bifurcation: a complex phase space study. J. Phys. A: Math. Theor. 41, 1-9 (2009)

[12] Hofbauer, J., Sigmund, K. Dynamical Systems and the Theory of Evolution. Cambridge University Press. Cambridge UK (1988)

[13] Hofbauer, J., Mallet-Paret, J., Smith, H. L. Stable periodic solutions for the hypercycle system. J. Dyn. and Diff. Eq. 3, 423-436
(1991)

[14] Campos, P.R.A., Fontanari, J.F., Stadler, P.F. Error propagation in the hypercycle. Phys. Rev. E 61, 2996-3002 (2000)

[15] Sardanyés, J. Error threshold ghosts in a simple hypercycle with error prone self-replication. Chaos, Solitons \& Fractals 35, 313-319 (2008)

[16] Silvestre, D.A.M.M., Fontanari, J.F. The information capacity of hypercycles. J. Theor. Biol. 254, 804-806 (2008)

[17] Attolini, C.S-O., Stadler, P.F. Evolving towards the hypercycle: A spatial model of molecular evolution Physica D 217, 134-141 (2006)

[18] Gray, R. M. Toeplitz and Circulant Matrices: A review (2001), avalaible at http://ee.stanford.edu/ gray/toeplitz.pdf

[19] Sardanyés, J. and Solé, R. V. Ghosts in the origins of life? Int. J. of Bif. and Chaos 16, 2761-2765 (2006).

[20] Duarte, J., Januáril, C., Martins, N., Sardanyés, J. On chaos, transient chaos and ghosts in single population models with Allee effects. Nonlinear Analysis: Real World Applications 13, 1647-1661 (2012).

[21] Ay, N., Erb, I. On the Notion of Linear Replicator Equations. Journal of Dynamics and Differential Equations, 17, 427451(2005)

[22] Doedel, E. J., Oldeman, B., et al. (2008) AUTO-07P: Continuation and Bifurcation Software for Ordinary Differential Equations. Department of Computer Science, Concordia University, Montreal, Canada. http://sourceforge.net/projects/auto-07p/

[23] Perko, L. Differential equations and dynamical systems. Third edition. Texts in Applied Mathematics, 7. Springer-Verlag (2001)

[24] Simó, C. On the analytical and numerical approximation of invariant manifolds. Les Méthodes Modernes de la Méchanique Céleste, D. Benest and C. Froeschlé, eds. 285-329 (1990) 